\title{
Esmaltes Digitales para un proceso de esmaltación y decoración totalmente digital
}

\author{
ESMALGLASS-ITACA GRUPO
}

\begin{abstract}
Esmalglass-Itaca Grupo ha sido galardonada por la Sociedad Española de Cerámica y Vidrio con un Alfa de Oro en su edición de 2011 por el siguiente trabajo de investigación: el desarrollo de una novedosa familia de esmaltes aplicados a través de sistemas digitales que permiten completar la innovación de proceso de fabricación industrial de baldosas cerámicas esmaltadas y decoradas digitalmente.
\end{abstract}

Palabras clave: Esmalte digital, Tinta trasparente, Inyección, Proceso digital.

\section{Digital Glazes for a totally digital glazing \& decorating process}

The Spanish Society of Ceramic \& Glass has awarded Esmalglass - Itaca Group with an "Alfa de Oro" on its 2011 edition, for the following investigation work: the development of a new range of glazes applied with digital systems that will allow to complete the innovation of the industrial manufacturing of glazed \& digitally decorated ceramic tiles.

Key words: digital glaze, clear ink, inkjet, digital process

\section{INTRODUCCIÓN.}

La decoración cerámica a través de la tecnología digital inkjet ha representado uno de los principales hitos de innovación acontecidos en la historia reciente de la cerámica plana industrial. Tras la aparición de los hornos monoestratos por rodillos y la importantísima aportación que tuvieron los sistemas de decoración por huecograbado, nos encontramos ahora con esta trascendente innovación en el sector cerámico.

Ha marcado historia porque sin duda es de las pocas innovaciones cerámicas capaces de ayudar al fabricante de pavimentos y revestimientos cerámicos a mejorar su posicionamiento en el mercado con un producto diferenciador, que permitirá contemporáneamente a tener plantas más flexibles y productivas sin incrementar los costes operativos, que ayudará a tener una mejor gestión de los almacenes, a poder hacer frente a la producción de partidas más cortas y/o personalizadas, en definitiva a poder afianzar las estrategias como empresa sobre la dimensión de negocio que más interese.

La tecnología inkjet ${ }^{[1]}$ nació en los años 70 como sistema de impresión en el marcado y codificación de productos. Con los años la tecnología prosperó hacia otros sectores, especialmente los de artes gráficas, y podemos decir que fue en el año 2000 cuando desembarca el primer prototipo de máquina industrial ${ }^{[2]}$ para el sector cerámico. Durante esta última década la tecnología ha evolucionado enormemente ${ }^{[3]}$. El continuo avance de cabezales ${ }^{[4,5]}$, de electrónica, de software y los continuos desarrollos de tintas han permitido una profunda consolidación de esta tecnología en el sector cerámico $^{[6]}$.
Durante estos 10 años que hemos convivido con esta tecnología podemos distinguir dos grandes etapas; una primera etapa hasta el año 2006 aproximadamente donde el sistema inkjet solo funcionaba con tintas solubles. En esta etapa se instalaron pocas unidades debido a que contaba con grandes limitaciones, especialmente con las relacionadas con los aspectos cromáticos, ya que las tintas solubles utilizadas limitaban mucho las posibilidades de realización de productos debido a su poca cromaticidad, inestabilidad e incluso coste. A partir de ese momento y con la introducción de las tintas pigmentadas se dio un salto cualitativo muy importante ${ }^{[7]}$. Las impresoras inkjet ya eran capaces con estos sets de tintas pigmentadas de imprimir gran parte del espacio cromático habitual al que está acostumbrado el sector y además a unos costes muy competitivos. Este salto fue si cabe amplificado con la entrada de nuevos componentes en el escenario competitivo; nuevos fabricantes de maquinaria y nuevos colorifícios; todo un colectivo de empresas obsesionado por poder dar al sector cerámico una solución óptima.

\section{VENTAJAS Y DESVENTAJAS ACTUALES DE LA TEC- NOLOGÍA INKJET CERÁMICO.}

Las ventajas de estos sistemas como la de decorar con alta definición de imagen y sin contacto, la posibilidad de adaptarse a cualquier tipo de formato y relieve, decorar el $100 \%$ de la superficie y la gran versatilidad gráfica por el mayor tamaño de los diseños han permitido sin duda tener avances muy significativos a nivel estético, pudiendo 
configurar líneas de producto para el segmento más alto del mercado. Pero esta tecnología no es sólo apta para competir con productos diferenciadores, también tiene muchísimas ventajas a nivel de procesos internos. Por ejemplo a nivel productivo la reducción de costes de desarrollo también es muy significativa; se reducen el número de pruebas, el tiempo invertido y la partida de costes de fungibles y consumibles típicos de las decoraciones convencionales es casi nula. También permite una mayor flexibilidad y rapidez en el cambio de modelaje, así como una reducción considerable en el número de tonos producidos, lo que agiliza todo el proceso productivo dentro de la planta. Además tras los análisis realizados entre diferentes clientes usuarios de esta tecnología, todas estas ventajas se obtienen sin incrementar los costes operativos por metro cuadrado y en muchas ocasiones incluso reduciéndolos notablemente. La simplificación de almacenes y la reducción de los mismos por la posibilidad de producir prácticamente ad-hoc y el hecho de reducir las referencias por tonos, permiten mejorar mucho el capital circulante de la compañía. En definitiva, son de las pocas tecnologías que satisface a diferentes estamentos dentro de la empresa usuaria como el Director Industrial, Director de Nuevos Productos, Director Comercial, Director Financiero y Director General, ya que todos ellos han encontrado aspectos muy positivos que han facilitado enormemente su trabajo.

Por otro lado, la tecnología digital también dispone de carencias o desventajas como son el menor rango cromático de las tintas respecto a los sistemas de decoración clásicos, la todavía poca robustez de estos sistemas para los ambientes cerámicos habituales o por ejemplo la falta de mayor productividad de las máquinas sin necesidad de perder definición o de hacer grandes inversiones duplicando las barras de impresión. El desarrollo cromático y el consumo de tinta depende de la composición química del esmalte y del ciclo de cocción, por lo tanto la composición de los esmaltes debe ser optimizada y las desviaciones en producción deben ser mínimas y controladas para no tener que retocar continuamente los archivos gráficos. Mención aparte es el hecho de que las tintas desarrolladas para esta tecnología han sido hasta la fecha tintas coloreadas que tenían como objetivo aportar de forma digital la gráfica y la estética de la baldosa cerámica desde un punto de vista estrictamente del color, obviamente fundamental para el desarrollo del producto.

\section{OBJETIVO DEL TRABAJO DE INVESTIGACIÓN.}

La cerámica no es solo color o gráfica, es también un juego de materias, de contrastes, de tactos, de brillos, de texturas, que le aporta esa diferenciación respecto a otros revestimientos. $Y$ ¿por qué no aprovechar las enormes ventajas que aporta la técnica digital para poder aplicar materia y así completar una línea de esmaltación completamente digital?

Por tanto en este punto se plantea el trabajo de investigación aquí descrito teniendo como objetivo el desarrollo de esmaltes que puedan aplicarse con sistemas digitales-que permitan decorar y esmaltar simultáneamente e incluso de forma sincronizada y así poder aportar efectos matéricos a la decoración cerámica con todas las ventajas de los sistemas digitales.

Aunque la verdadera innovación que se pretende conseguir es una innovación de proceso ya que de esta forma se cierra el círculo para producir por primera vez un azulejo íntegramente con tecnología digital, extendiendo al resto de materiales cerámicos las mismas y numerosas ventajas que ofrece la digitalización para el color y la gráfica.

\section{RESULTADOS DEL TRABAJO DE INVESTIGACIÓN.}

Tras varios años de estudios con personal experto en inyección y en esmaltes nanométricos, se presenta la nueva familia de Esmaltes Digitales DPG (Digital Printing Glaze). Con esta revolucionaria solución digital, se logra por primera vez un azulejo producido íntegramente con tecnología digital. Asimismo se consigue realizar desde el engobe inicial, pasando por la decoración, hasta llegar a la protección final de azulejo.

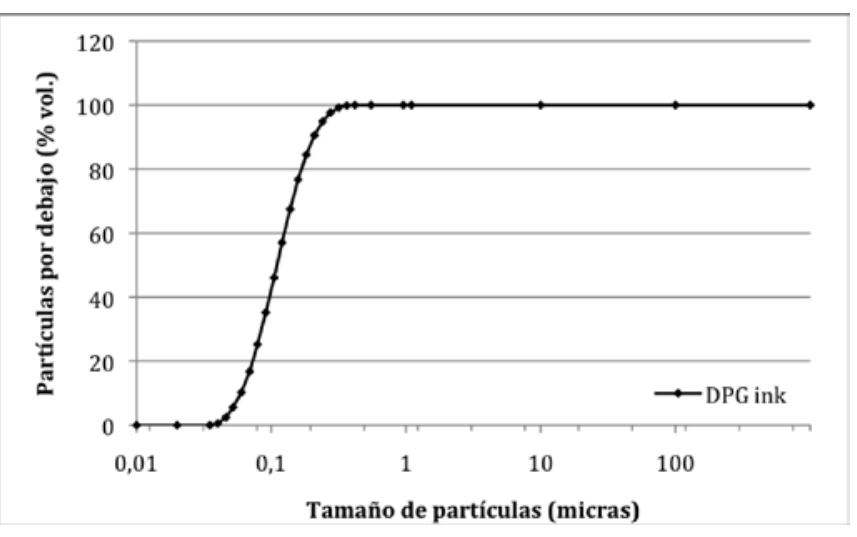

Distribución granulométrica de un esmalte DPG.

Esta familia de Esmaltes Digitales DPG está especialmente diseñada y desarrollada para su aplicación digital a través de cabezales de inyección DOD (Drop On Demand), ofreciendo nuevas posibilidades al proceso digital cerámico.

La familia consta actualmente de un esmalte digital blanco opaco y un esmalte digital transparente, ambos válidos para todas las tecnologías de fabricación existentes.

- Esmalte Digital DPG Blanco Opaco. Diseñado para utilizarse de dos maneras: bien como tinta blanca para crear decoraciones, por ejemplo vetas sobre fondos oscuros, o bien como base del azulejo o base de alguna tinta digital para realzar su color.

- Esmalte digital DPG Transparente. Diseñado para crear la protección final de azulejo y crear por primera vez y de manera totalmente digital un diseño de cubierta perfectamente sincronizado con el diseño de la base.

Desde el punto de vista técnico la serie de Esmaltes Digitales DPG contiene partículas nanométricas cuya distribución granulométrica estrictamente controlada confiere una excelente estabilidad física además de prestaciones óptimas en los cabezales de inyección, variables críticas en este trabajo de investigación.

Asimismo, con el paso de la escala micrónica a la nanométrica, se ha estudiado ampliamente los cambios de propiedades de los materiales tanto de naturaleza vítrea como cristalina, de manera a diseñar y optimizar novedosas 


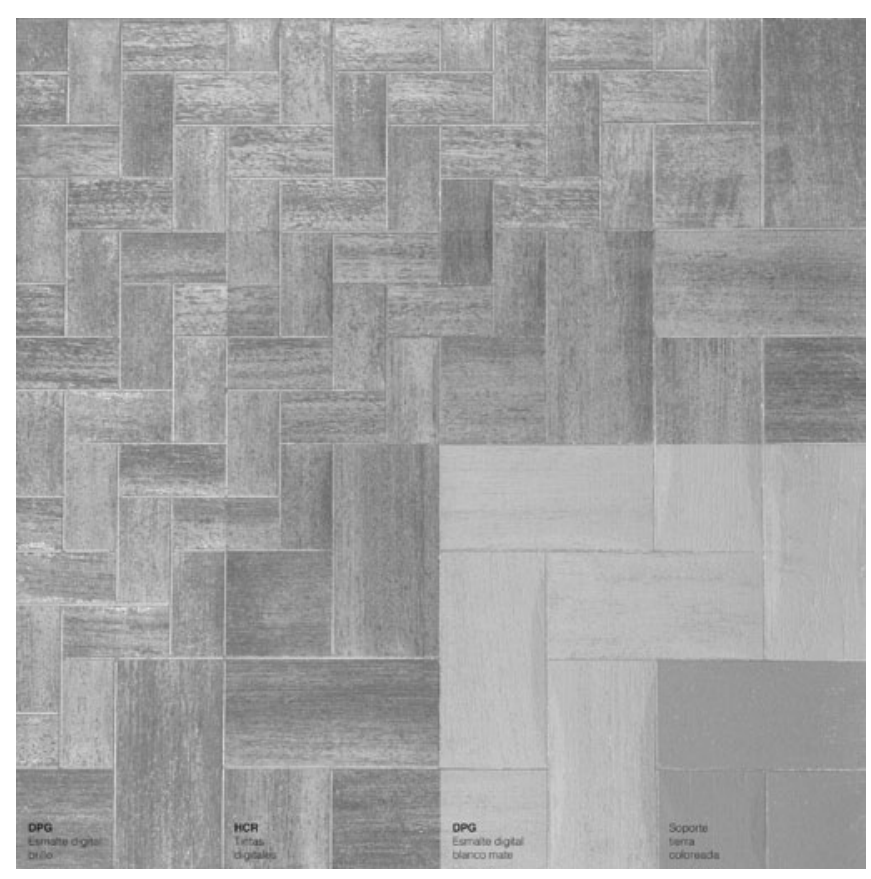

Desglose de aplicaciones (de derecha a izquierda) de la pieza anterior.

composiciones con buenas propiedades técnicas y estéticas requeridas para los productos cerámicos decorados. Además, también se garantiza el máximo desarrollo cromático de las tintas pigmentadas inkjet Esmalglass-Itaca.

La formulación de los Esmaltes Digitales DPG ha sido rigurosamente ajustada por una parte para cumplir con las especificaciones de las impresoras industriales cerámicas, y por otra para ser respetuosas con el medio ambiente.

\section{Ventajas de la esmaltación digital y de la decoración con esmaltes digitales.}

\section{Estéticas.}

Aplicación digital sin contacto para esmaltar bajo relieves Esmaltar hasta el borde de la pieza sin acúmulos

Versatilidad en el diseño de la esmaltación

Esmaltación con alta calidad de impresión

Reproducibilidad de otras técnicas (doble carga,

doble prensa,...)

Poca repetitividad en la esmaltación de grandes formatos

Rápido desarrollo de modelaje

Lo que va a permitir:

Alto grado de diferenciación

Grandes posibilidades de contratipo

Nuevas posibilidades para los diseñadores

Sincronizar la esmaltación con relieves y diseños

\section{Productivas.}

Eliminación de cuellos de botella en la preparación de esmaltes

Uso garantizado de esmaltes de alto desarrollo cromático Eliminación de fases de preparación y control previo de esmaltes

Líneas de esmaltar mucho mas cortas
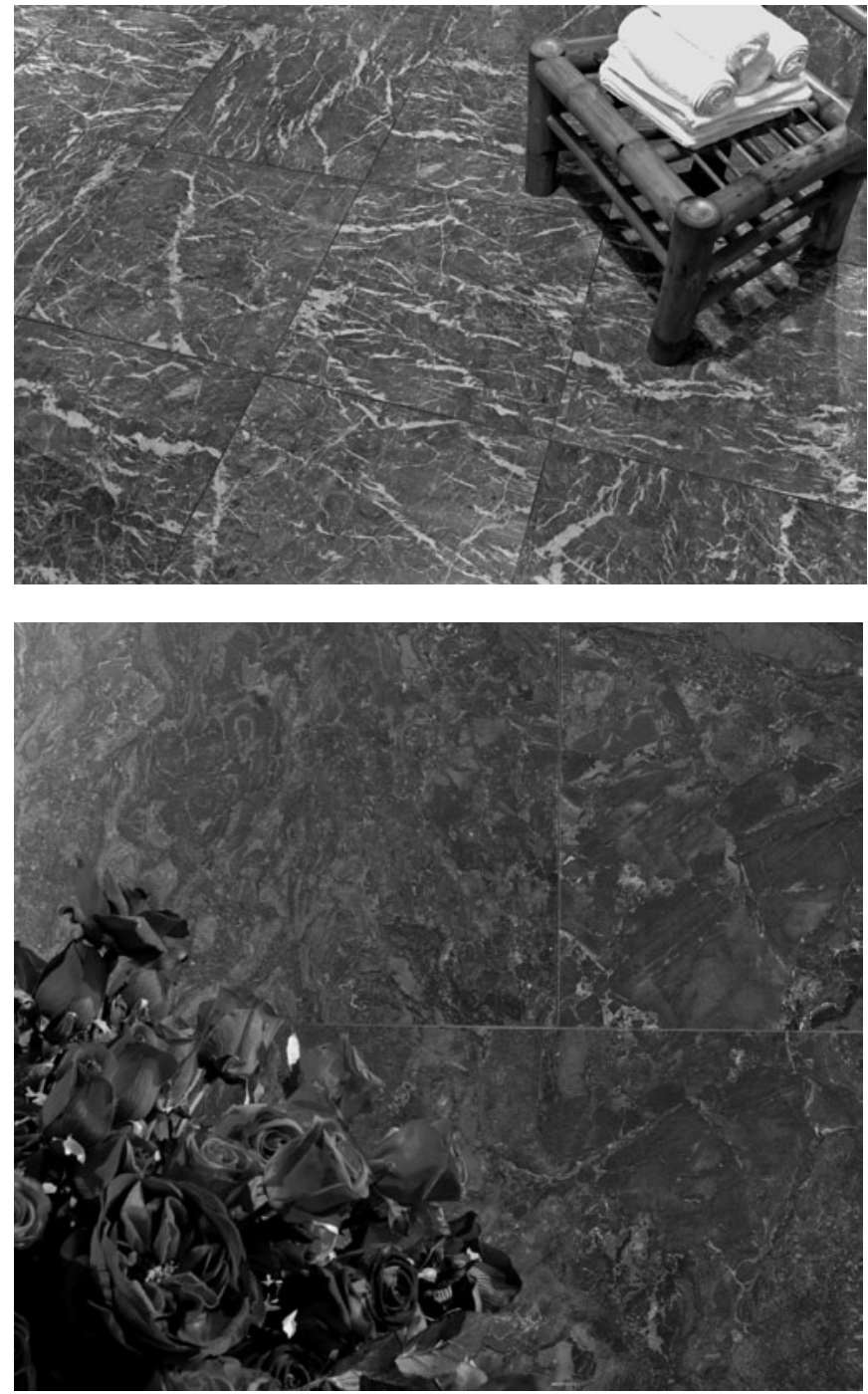

Ejemplo de piezas realizadas con la aplicación de Esmalte Digital DPG Blanco Opaco.

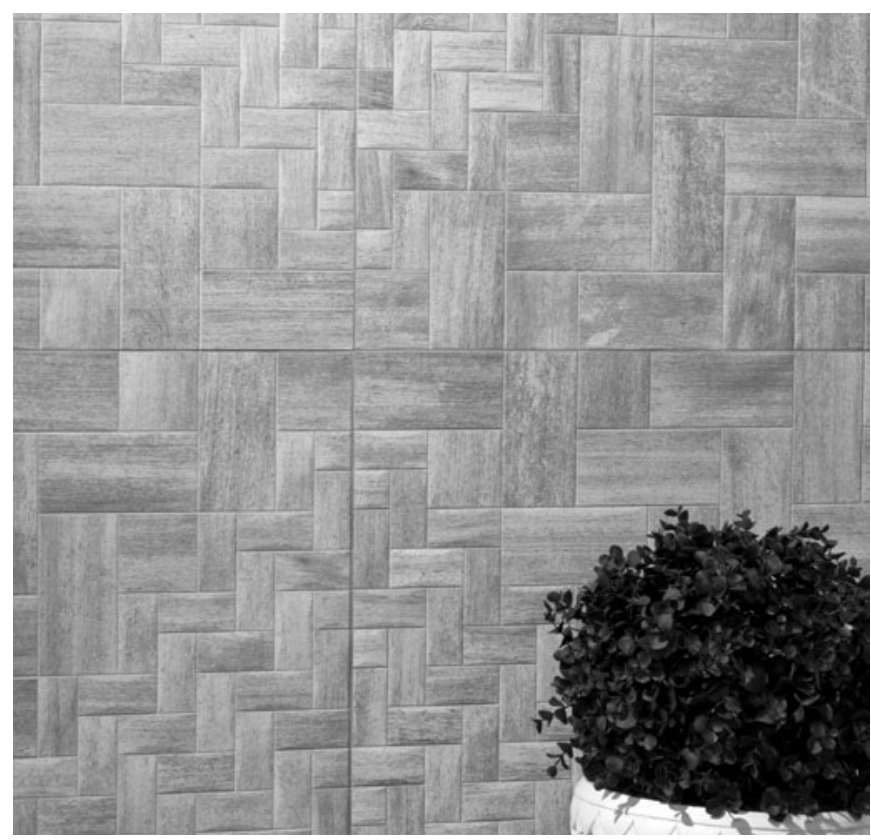

Ejemplo de piezas realizadas con la aplicación de Esmalte Digital DPG Blanco Opaco y Transparente. 
Eliminación de los clásicos problemas en inkjet

por evaporación de agua en los esmaltes

Reducción de tiempos muertos por ajustes y limpiezas

Reducción de la defectología, óptimo control reológico

Eliminación de picos de producción de esmaltes

Elevada Flexibilidad Productiva

Aumento de la Productividad

\section{Logísticas.}

Capacidad de hacer partidas ajustadas

Reducción y simplificación de stocks

Reducción en el $\mathrm{n}^{\mathrm{o}}$ de esmaltes a utilizar

\section{Económicas.}

Eliminación de fungibles

Eliminación de espacios; línea de esmaltar y sección

prep. Esmaltes

Reducción de picos de esmaltes

Reducción costes preparación y control de esmaltes

Mejora en la eficiencia y eficacia del desarrollo de producto

Incremento de los porcentajes de primera calidad

Mayor productividad por eliminación de cuellos de botella

en:

Preparación de línea

Ajustes de línea

Reducción de los tiempos de limpieza

\section{CONCLUSIONES.}

- El grupo Esmalglass-Itaca ha desarrollado una novedosa familia de esmaltes que, aplicados a través de sistemas digitales, permiten innovar de nuevo en el proceso productivo de la fabricación de baldosas cerámicas, complementando la actual decoración inkjet con la esmaltación digital. Por lo tanto, se logra por primera vez un azulejo producido íntegramente con tecnología digital, extendiendo al resto de materiales cerámicos las mismas y numerosas ventajas que ofrece la digitalización para el color y la gráfica.

- Latecnología digital inkjetrepresenta uno delos principales hitos de innovación en la historia reciente de la cerámica industrial. Durante esta última década, esta tecnología ha evolucionado continuamente con importantes avances que han permitido su consolidación en el sector cerámico. Las numerosas ventajas de la decoración digital industrial con tintas pigmentadas, como el aumento del porcentaje de primera sin incremento de costes operativos por metro cuadrado y en muchas ocasiones incluso con reducción notable, han aportado aspectos muy positivos a la gestión global de la empresa.
- Las tintas desarrolladas para esta tecnología han sido hasta la fecha tintas coloreadas que tenían como objetivo aportar de forma digital la gráfica y la estética de la baldosa cerámica. Pero la cerámica no es solo color o gráfica, es también un juego de materias que le aporta esa diferenciación respecto a otros revestimientos. Los innovadores esmaltes digitales permiten decorar y esmaltar simultáneamente e incluso de forma sincronizada y así poder aportar efectos matéricos a la decoración cerámica obteniendo un mayor grado de diferenciación. Asimismo se consigue realizar desde el engobe inicial, pasando por la decoración, hasta llegar a la protección final de azulejo. Se han obtenido resultados satisfactorios en cuanto a la estabilidad y comportamiento de los esmaltes, gracias a su naturaleza nanométrica, para ser aplicados en tecnologías digitales actuales de tipo inkjet.

- Esmaltar y decorar digitalmente para poder aplicar color, gráfica y materia al producto cerámico, no solo mantiene las mismas ventajas reconocidas pero también aporta nuevas ventajas como, entre tantas, la sincronización de la esmaltación con relieves y diseños, líneas de esmaltar mucho más cortas y la eliminación de los clásicos problemas en inkjet por evaporación de agua en los esmaltes.

- La innovación de proceso que Esmalglass-Itaca Grupo presenta y que ha sido galardonada con el Alfa de Oro de la SECV en su edición de 2011 permitirá aportar nuevas herramientas en el desarrollo de producto, nuevos conceptos de lay-out de las líneas de fabricación y nuevos conceptos de gestión de las plantas productivas cerámicas y de su propia logística de comercialización, en definitiva, nuevos argumentos para hacer más competitivo si cabe el sector de baldosas cerámicas frente a otros sectores de recubrimientos de superficies.

\section{BIBLIOGRAFÍA.}

1. R.G. Sweet (1971) "Fluid droplet recorder", US 3.596.275.

2. J.V. Tomás Claramonte (2001) "Dispositivo para decoración de baldosas cerámicas", ES 2.152.167.

3. I. Hutchings (2010) "Impresión por chorro de tinta para la decoración de baldosas: tecnología y oportunidades", QUALIcer'10.

4. M. Alexander (2008) The Xaar guide to single pass printing, Technical White Paper, www.xaar.com

5. M. Schoeppler (2009) "The Paradigm Shift to Single Pass", presentado en IMI's 17th Annual European Ink Jet Printing Conference (Barcelona).

6. M. Alexander (2010) The Xaar guide to digital printing in the ceramics sector, Technical White Paper, www.xaar.com

7. Esmalglass-Itaca (2007) "Esmalglass-itaca, proveedora de soluciones globales para los sistemas de decoración digital"; Cerámica Información n'341, pp.65-69. 